\title{
Realidade Virtual Como Ferramenta Complementar Para Reabilitação Da Paralisia Do Membro Superior Distal Causada Por AVC
}

\author{
Lucas Oliveira \\ Núcleo de Computação \\ Instituto Federal Goiano \\ Morrinhos Goiás Brasil \\ lucas.keterson@estudante.ifgoiano.edu.br
}

\author{
Alexandre Silva \\ Núcleo de Computação \\ Instituto Federal Goiano \\ Morrinhos Goiás Brasil \\ alexandre.silva@ifgoiano.edu.br
}

\begin{abstract}
RESUMO
Os métodos convencionais de reabilitação em pacientes que sofreram acidente vascular cerebral (AVC) usam na maioria das vezes metodologias monótonas, podendo reduzir a carga motivacional do paciente e de certa forma minimizar os avanços da proposta de recuperação. Assim, surge a motivação de investigar a utilização de técnicas de Realidade Virtual (RV) para reduzir esta problemática. Portanto, esta pesquisa tem como abordagem inicial desenvolver um protótipo funcional $\mathrm{e}$ operacional capaz de atuar de forma complementar ao processo de reabilitação da paralisia do membro superior distal, causada por AVC. Para a concretização desta proposta foi utilizado o sensor Leap Motion Controller (LMC) para captar ações das mãos e rastrear as mesmas de forma precisa, e simultaneamente, integrado ao uso do motor gráfico Unity3D para desenvolver mecânicas para o sistema. Neste cenário, é importante garantir que o protótipo implementado esteja com diretrizes de funcionamento e operações corretas, para que em pesquisas posteriores ele possa ser usado com acompanhamento de um terapeuta ou de forma isolada pelo paciente em espaço doméstico, avaliando os ganhos obtidos por esta solução, podendo desta forma tornar-se um instrumento complementar distinto no processo de reabilitação convencional. Por fim, após testes de funcionalidade foi possível constatar que o sistema desenvolvido se comportou de forma eficiente, executando as funções para as quais foi desenvolvido.
\end{abstract}

\section{PALAVRAS CHAVE}

Leap Motion Controller; Reabilitação; Realidade Virtual.

\section{Introdução}

A fisioterapia neuromuscular é utilizada para reabilitação daqueles que sofreram um AVC, esta se torna intensiva e cara, o que exige um tratamento eficaz relacionado a custo-benefício. Os custos com medicamentos com pacientes passam a faixa dos bilhões por ano [1].

Os tratamentos são tão caros pelo motivo do AVC ser o distúrbio neurológico mais frequente do mundo. Este distúrbio ataca principalmente as funções motoras afetando a vida dos pacientes. Para uma melhor evolução do tratamento torna-se necessário atividades relacionadas as necessidades dos pacientes, devido a isso surgem novas estratégias de reabilitação [15].

Os ambientes virtuais surgem como estratégia e vem ganhando cada vez mais força no ambiente terapêutico, porém em vários casos estes ambientes não possuem métricas de reabilitação[2].

Devido a constatação dessas dificuldades, surge a seguinte problemática: Como minimizar as dificuldades enfrentadas no processo de reabilitação da paralisia do membro superior distal causada por AVC?

Sendo assim está pesquisa tem como objetivo geral desenvolver um protótipo baseado em técnicas de RV, para elaboração de mecânicas de reabilitação que promova um sistema complementar funcional para recuperação do membro superior distal.

Tendo como objetivos específicos:

a) Desenvolver um protótipo capaz de ser executado em computadores de desempenho comum.

b) Apresentar ao terapeuta o desempenho do paciente ao utilizar o protótipo.

Dessa forma, justifica-se nessa pesquisa as vantagens que um ambiente virtual aplicado na reabilitação proporciona como: movimentação baseada na experiência diferenciada, estímulos multissensoriais, contribuição na consolidação da RV como instrumento de reabilitação.

\section{Referencial teórico}

O AVC é uma doença que ataca o sistema nervoso central como resultado de um distúrbio na circulação cerebral. Existem dois tipos de AVC, isquêmico e hemorrágico, que resultam na danificação da função cerebral[3].

No Brasil, as pessoas com deficiência têm direito de acesso à saúde assegurado na constituição de 1988. Em 2008, a Organização Mundial de Saúde cria uma política que dá direitos de acesso a reabilitação para pessoas com deficiência. Existem cerca de 2.5 milhões de pessoas com deficiência física no Brasil, porém menos de um quinto frequentam algum tipo de serviço de reabilitação. Uma das causas do pequeno número de pessoas que recorrem a reabilitação deve-se a falta de orientação e encaminhamento no momento de alta hospitalar, deixando os pacientes inseguros e desassistidos ao retornarem ao domicílio [4]. 
SVR2021' 18, October, 2021, Gramado, Rio Grande do Sul, Brazil

Os sobreviventes afetados por AVC enfrentam diversas dificuldades. Estes podem manifestar sequelas que prejudicam a qualidade de vida, desde falhas nas funções físicas a prejuízos cognitivos. Cerca de 50\% dos sobreviventes têm o membro superior acometido por paralisia. Esta paralisia é trabalhada na reabilitação conduzida por terapeutas, que tem como objetivo o alcance da independência e autonomia[5].

Para o entendimento da reabilitação física em pacientes com diagnóstico de hemiparesia, é necessário definir este tópico, considerando que: etimologicamente, reabilitação significa reparar ou restabelecer uma condição.

Após realizar as abordagens anteriores e entender o que o sobrevivente de AVC enfrenta agora é possível dar início aos tipos de tratamentos convencionais e conhecidos usados para o desenvolvimento do protótipo.

A Cinesioterapia é um recurso fisioterapêutico que atua na prevenção de deformidades e na reeducação neuro-motora. As diversas técnicas de Cinesioterapia objetivam diminuir a hipertonia, fortalecer a musculatura, manter a amplitude de movimento, proporcionar estimulação sensorial e proprioceptiva, utilizando postura e exercícios funcionais [6].

O Teste de Caixa e Blocos foi criado para ser um teste para pessoas com deficiência física. Permite avaliar e medir a destreza manual de pacientes com problemas neuromusculares[8].A dinâmica de execução baseia-se em uma caixa de madeira com uma divisória ao meio, e conta com múltiplos blocos de cores diferentes. O paciente deve carregar um bloco de cada vez de um compartimento a outro, este só pode soltar um bloco uma vez que a ponta de seus dedos esteja posicionada totalmente do outro lado da divisória. Se o bloco cair, o paciente pode pegá-lo e continuar a tarefa sem nenhum tipo de penalidade[7].

Durante o método tradicional de reabilitação existem diversos fatores que dificultam esse processo, no contexto desta pesquisa merecem destaque:

Acesso: Estima-se que a carência de acesso à informação e ao sistema de saúde pode aumentar a chance de acometimento por AVC. Quanto maior o nível de formação, maior o conhecimento sobre a doença, seus fatores de risco e menor índice de comportamentos de risco[9];

Custos: Devido ao baixo nível de escolaridade e poder econômico dos pacientes atendidos em hospitais públicos, sabe-se que este tem pouca compreensão sobre o tratamento e prevenção do AVC. Isso pode contribuir para que este tipo de acidente esteja em primeiro lugar nas causas de óbitos na maioria dos estados do Brasil. Um estudo mostrou que os gastos médios diários de um paciente internado com complicações passam dos U\$100.00[10].

Pode-se dizer que levando esse valor em consideração para cada paciente internado por causas do AVC, se somados, no final é possível observar que os gastos são extremamente elevados.

\subsection{Realidade Virtual}

L. Oliveira \& A. Silva

RV é uma tecnologia computacional que oferece a ilusão ao usuário de estar imerso em um espaço tridimensional, com a habilidade de interagir com este espaço 3D[11].

A RV é composta por três princípios básicos:

A. Interatividade - Relacionada com a capacidade do computador detectar as ações do usuário e reagir instantaneamente, modificando aspectos da aplicação [12].

B. Envolvimento - Ligado ao grau de motivação para o engajamento de uma pessoa em determinada atividade [13] [18].

C. Imersão - Sensação ou sentimento de estar dentro do ambiente [12].

A RV tem o poder de passar a sensação de estar dentro de um ambiente virtual, e a capacidade de permitir ao usuário a interação com este ambiente de maneira intuitiva e natural[14].

\section{Trabalhos Relacionados}

É possível encontrar diversos trabalhos de aplicações de jogos sérios na reabilitação da hemiplegia causada por AVC. Para este trabalho foram selecionados três trabalhos que correlatam com o tema abordado. Estes trabalhos foram analisados com base em critérios relevantes, com intuito de posicionar, verticalizar e implementar aspectos importantes como foco da aplicação.

Soares [15] tem como objetivo em seu trabalho, avaliar a aplicabilidade do LMC combinado com a reabilitação pós AVC do membro superior.

Foram escolhidos três pacientes, estes foram avaliados por três dias consecutivos usando técnicas de reabilitação comuns e a aplicação do jogo Playground3D do próprio LMC, e no último dia aplicou-se uma ficha de avaliação. Os treinamentos eram realizados em ambos os membros, parético e não parético.

Os resultados foram positivos e demonstraram um treinamento promissor já que a ferramenta proporcionou a curto prazo uma diminuição do limiar motor cortical, melhores desempenhos nos testes de habilidade manual e óculo-manual e foi bem recebido pelos pacientes testados.

Já Cyrino [16] propõe um jogo sério customizável e adaptável de RV para reabilitação pós AVC. Este é uma aplicação desktop composta por quatro camadas intercomunicáveis, sendo elas: painel de configuração, o jogo, banco de dados e interface.

Para testes foram selecionados seis pacientes com AVC isquêmico. $\mathrm{O}$ treinamento destes mudava de dificuldade gradativamente pelo terapeuta cada vez que o voluntário mostrasse facilidade em completar as fases do jogo.

Os resultados apresentaram uma grande evolução dos pacientes até a terceira sessão, após isso houve uma queda devido a modificação dos parâmetros de dificuldade para os pacientes que se adaptaram bem. Por meio de um questionário aplicado aos pacientes, estes expressaram satisfação com o ambiente virtual.

Por fim, Balista [2], teve como objetivo construir um jogo sério para desktops usando o sensor de captura do Xbox 360, o Kinect. 
Realidade Virtual como ferramenta complementar para reabilitação da paralisia do membro superior distal causada por AVC.

A aplicação é composta por três jogos que trabalham membros diferentes, o jogo número um trabalha os membros inferiores e o equilíbrio, o jogo número dois trabalha a cognição e capacidade motora e o último trabalha os membros superiores. $O$ trabalho não apresenta resultados em relação a evolução dos pacientes, mas Balista expões as métricas de avaliação usadas, sendo estas, pontuação, distância percorrida, tempo e amplitude dos movimentos.

Com base nos trabalhos abordados, foi possível observar como os jogos, até aqueles não projetados com intuito de reabilitação, trouxeram ganhos motivacionais e físicos positivos aos usuários.

\section{Descrição do protótipo}

O sistema proposto trata-se de um ambiente virtual com recursos tridimensionais, que tem a finalidade de representar métodos de reabilitação convencionais de forma virtual e com métricas de medição de tempo e pontos.

O protótipo desenvolvido utiliza o motor gráfico Unity3D, em conjunto com kit de desenvolvimento do LMC, tornando-se possível realizar a captura e rastreamento dos movimentos das mãos em tempo real e com precisão.

O ambiente virtual permite estabelecer tempo de sessões de treinamento, mensurar o tempo gasto para obtenção dos objetivos. Este também fornece um sistema de pontuação gráfica por meio de um gráfico de linhas. O protótipo realiza o envio da pontuação de cada sessão para o terapeuta.

Ao iniciar, o usuário encontrará uma interface na qual deve escolher se irá desempenhar o papel de terapeuta ou de paciente. Uma vez escolhido paciente é apresentado um menu, com diversas funcionalidades. Neste menu, o paciente pode visualizar os tutoriais de cada narrativa, escolher um exercício para realizar ou visualizar os gráficos de desempenho de cada exercício.

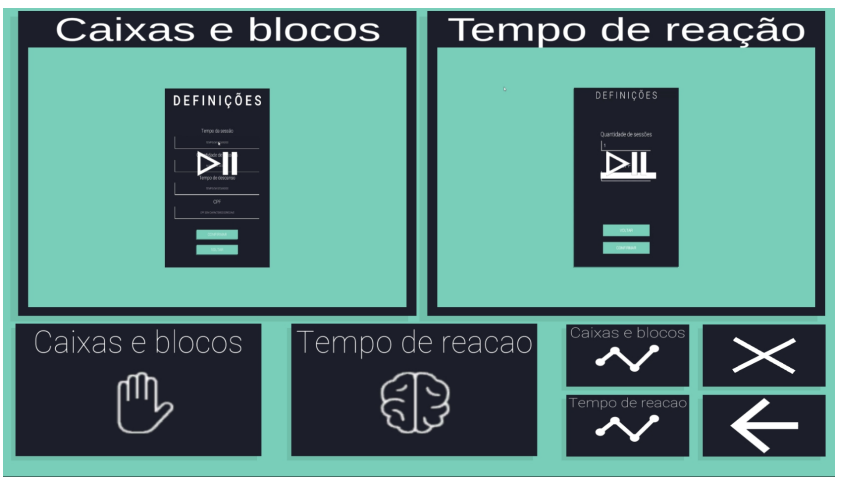

Figura 1: Menu do paciente

A primeira narrativa do jogo, consiste em um espaço tridimensional com quatro caixas, de cores diferentes, localizadas a frente do usuário. Blocos de cores correspondentes a cada caixa aparecem na cena. $\mathrm{O}$ usuário então deve pegar o bloco e levar este
SVR2021' 18, October, 2021, Gramado, Rio Grande do

Sul, Brazil

até a caixa com a cor correspondente, uma vez colocado dentro da caixa a pontuação é somada. Se a caixa for da cor incorreta o bloco é reposicionado. A narrativa tem um tempo de descanso a cada vez que o tempo de sessão chega a zero,evitando causar fadiga e cansaço ao paciente.

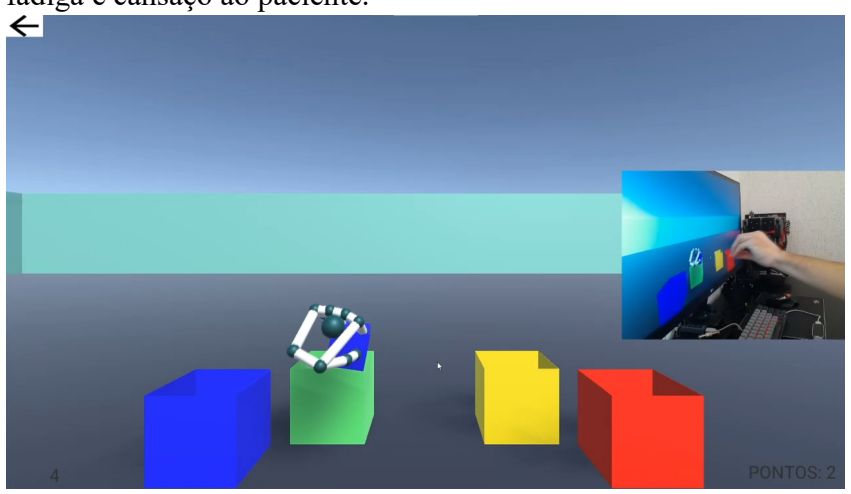

Figura 2: Primeira narrativa "Caixa e Blocos"

De forma resumida, o dispositivo de captura LMC realiza a captura dos movimentos, estes são interpretados pelos drivers do dispositivo e enviados ao motor gráfico Unity3D, em tempo real e com alta precisão. Na Unity3D os movimentos serão usados para conclusão de objetivos que a narrativa do jogo exigir. Após o término da sessão os exercícios concluídos irão gerar pontos, os quais serão enviados pela Unity3D para o Google Sheets.

\section{Implementação}

Para o desenvolvimento do protótipo foram usadas um total de cinco tecnologias, sendo elas, Blender, Unity3D, Visual Studio 2019, LMC e Google Sheets.

O Blender que é um software de modelagem destinado a trabalhos gráficos e de código aberto, foi utilizado para modelagem dos cubos e das caixas da primeira narrativa, Caixa e Blocos.

A Codificação do sistema foi realizada usando o ambiente de desenvolvimento integrado que acompanha a Unity3D, o Visual Studio 2019. Esta foi realizada na linguagem C\# que atualmente é a única linguagem de programação aceita pela Unity3D.

Para a captura dos movimentos foi utilizado o sensor LMC devido a sua precisão e ao seu tamanho reduzido. Para o uso do sensor com a Unity3D é necessário o uso do Kit de Desenvolvimento de Software (SDK) que traz algumas cenas pré configuradas para uso.

O Google Sheets que se trata das folhas de cálculo do Google, serviu como banco de dados no protótipo. Nele foram armazenadas as pontuações de cada sessão de cada paciente conforme dados solicitados na configuração.

Por fim, a game engine de criação de mídia interativa, foi utilizada para juntar todas as tecnologias citadas anteriormente em narrativas interativas com mecânicas bem definidas. 
SVR2021’ 18, October, 2021, Gramado, Rio Grande do Sul, Brazil
L. Oliveira \& A. Silva

\section{Testes e Resultados}

Para testes, foi utilizado o método de caixa preta, o qual é um teste funcional, pois este se preocupa com a função que o programa desempenha, desconsiderando a maneira que foi implementado. Este teste pode ser classificado como um teste dinâmico já que o software é executado enquanto testado e o analista não tem conhecimento da estrutura interna da implementação do produto testado [17]. Para este trabalho, tornase de suma importância produzir um sistema livre de falhas e erros, para amenizar qualquer transtorno que este possa causar ao paciente, já que este pode considerar a má execução dos exercícios ao invés de considerar que o sistema não está executando de forma correta.

Durante os testes foram analisadas funções que recebem maiores quantidades de entradas, sendo estas, configurar atividade e realizar atividade.

Para validação do teste de configuração de atividade, foi necessário à validação das entradas que a tela de configurações recebe. Estas são tempo de sessão, quantidade de sessões, tempo de descanso, Cadastro de Pessoa Física (CPF) e confirmar. Para todos os campos que tem como entrada um valor inteiro, foram testados números negativos, o número zero, números que fossem maior que o limite do tipo inteiro, letras e símbolos. Como esperado o sistema se comportou de forma correta, aceitando apenas valores positivos e maiores que zero, porém não alcançou o limite do inteiro, aceitando apenas números menores que 99999. O campo CPF também se comportou como esperado, aceitando apenas números de onze dígitos, que fossem validos. Por último, o botão de confirmação das entradas, também se comportou corretamente, iniciando a atividade após a confirmação de todos os campos, e esperando cinco segundos antes da inicialização da cena.

Por outro lado, foi avaliado a execução do exercício. Neste avaliou-se os movimentos das mãos e as respostas do sistema a interação do usuário com o ambiente. Os casos avaliados foram, fechar a mão, abrir a mão, colocar o bloco na caixa e apertar a tecla espaço. Os casos testados referentes a movimentos das mãos, responderam de forma correta, segurando o bloco quando esperado e soltando o mesmo quando a mão fosse aberta. O sistema executou a soma da pontuação de forma correta ao colocar os blocos dentro das caixas, assim como resetou a posição do bloco ao apertar a tecla espaço.

Pode-se afirmar que o sistema concluiu todas as tarefas testadas de forma correta, e não apresentou erros durante a execução e testes. Assim o sistema passou de forma eficaz pelo teste de caixa preta.

\section{Conclusões}

Esta pesquisa teve como objetivo geral o desenvolvimento de um protótipo para auxílio da reabilitação da paralisia do membro superior causado pelo AVC, com intuito de promover uma possível ferramenta complementar no tratamento gerando autonomia para o paciente a partir do momento em que o terapeuta permita o uso do protótipo, além da redução de custos no tratamento a partir do uso de um sensor de baixo custo, sendo este o LMC.

Durante o desenvolvimento e testes do protótipo, o sensor de captura se comportou bem, conseguiu rastrear os movimentos com precisão durante a maior parte do tempo. Este teve dificuldades em rastrear nos primeiros momentos em que as mãos eram inseridas no campo de visão do sensor. Para solucionar o problema, foi colocado um contador de 5 segundos após a configuração da atividade "Caixa e Blocos", por ser a cena em que o erro causava mais impacto.

Devido ao software ser dirigido a pessoas que já sofrem com a paralisia causada pelo AVC este deve funcionar de forma precisa e correta, para evitar mais transtornos aos pacientes, e que a experiência de uso seja fluida e confortável podendo trazer assim melhores resultados e maior motivação na hora de usar a ferramenta. Por esse motivo foram realizados teste de caixa preta com o protótipo com o objetivo de verificar aspetos funcionais do sistema, e encontrar erros relacionados ao uso.

Após os testes da aplicação, foi possível verificar que o protótipo se comportou de forma correta e prevista. A aplicação conseguiu rastrear as mãos com alta precisão na maior parte do tempo, além de disponibilizar de forma quantitativa o desempenho atingindo mostrando para o usuário um histórico em forma de gráfico para um acompanhamento visual de seu desempenho. A aplicação também possibilita o armazenamento dos dados necessários de forma correta no Google Sheets, proporcionando ao terapeuta uma forma de acompanhamento do desempenho do paciente.

\section{Trabalhos Futuros}

Como o LMC teve dificuldades em rastrear as mãos em alguns momentos, para testar uma proposta diferente e que possa trazer benefícios, propõe-se a adaptação do protótipo para que este possa ser usado em conjunto com sensores convencionais.

Em função da necessidade do uso de um novo sensor, torna-se necessário à validação por comparativa dos sensores. Deseja-se comparar a precisão dos sensores e verificar qual destes teria melhor desempenho em conjunto com o sistema desenvolvido.

Devido ao estado atual da pandemia do COVID-19, foi descartado a possibilidade de realizar teste com usuários, por este motivo propõe-se como trabalho futuro os testes com usuários afetados por paralisia pós AVC, seguindo as normas de saúde e após protocolar e receber anuência do comitê de ética.

Estes testes visam verificar se o paciente irá obter algum tipo de evolução dos movimentos usando o protótipo desenvolvido nesta pesquisa. 


\section{Realidade Virtual como ferramenta complementar para reabilitação da paralisia do membro superior distal causada por AVC.}

Outro trabalho futuro bastante relevante diz respeito a procurar novas soluções que possam ser implementadas na ferramenta desde o ponto de vista do paciente, assim como também do ponto de vista do terapeuta podendo assim sempre atualizar a ferramenta com nova funcionalidades que possam ajudar ambos ou inclusive com novas narrativas.

Também se propõe a implementação de um sensor estereoscópico de alta imersão, óculos de RV, para realizar estudos em como a imersão maximizada do paciente pode afetar nos resultados dos treinamentos realizados na aplicação.

Em relação ao armazenamento de dados e análises dos mesmo, torna-se relevante a implementação de um banco de dados, assim como uma API para divulgação colaborativa de novos usuários e terapeutas. Por fim, criar um sistema de cadastro e login tanto para terapeutas como para pacientes.

\section{REFERÊNCIAS}

[1] Aviv Elor, Mircea Teodorecu, Sri Kurniawan. 2018. Project Star Catcher: A novel immersive virtual reality experience for upper limb rehabilitation. ACM Transactions.

[2] Vania Gabriella Balista. 2013. Sistema de realidade virtual para avaliação e reabilitação de déficit motor. Proceedings do XII Simpósio Brasileiro de Games e Entretenimento Digital.

[3] Diana Cancela. 2008. Acidente vascular cerebral-classificação, principais consequências e reabilitação. O portal do Psicólogo, Portugal.

[4] Emanuele Farencena Franchi et al. 2017. Prevalência de pessoas com deficiência física e acesso ao serviço de reabilitação no Brasil.. Cinergis, v. 18, n. 3, p. 169 .

[5] Thamyres Pereira Alves et al. 2020. Prática Mental, Terapia Ocupacional e Reabilitação no Acidente Vascular Cerebral. Revista Neurociências, v. 28, p. 125.

[6] Camila Rosa, Carolina Rosa, Denise Campos. 2013. Técnicas para reabilitação neurológica de hemiparéticos.

[7] Maria Fernanda Mendes et al. 2001. Teste de destreza manual da caixa e blocos em indivíduos normais e em pacientes com esclerose múltipla. Arquivos de Neuro-Psiquiatria, v. 59.

[8] Renata Guimarães, Silvana Maria Blascovi-Assis. 2012. Uso do teste caixa e blocos na avaliação de destreza manual em crianças e jovens com síndrome de Down. Revista de Terapia Ocupacional da Universidade de São Paulo, v. 23, n.

[9] Beatriz Abramczuk, Edlaine Villlela. 2009. A luta contra o AVC no Brasil. ComCiência, n. 109.

[10] Afonso Carlos Neves et al. 2002. Custos do paciente com acidente vascular cerebral no setor de emergência do Hospital São Paulo. Revista Neurociências, v. 10, n. 3 .

[11] Christine M. Byrne. 1996. Water on tap: The use of virtual reality as an educational tool. Doctoral dissertation, University of Washington.

[12] Romero Tori, Cláudio Kirner, Robson Siscoutto. 2006. Fundamentos e tecnologia de realidade virtual e aumentada. Belém: VIII Symposiun on Virtual Reality.

[13] Antônio Valério Netto, Liliane dos Santos Machado, Maria Cristina F. Oliveira 2002. Realidade Virtual - Fundamentos e Aplicações. Florianópolis-SC: Visual Books.

[14] Alexandre Cardoso, Cláudio Kirner, Edgard Lamounier, Judith Kelner. 2007. Tecnologias para o desenvolvimento de sistemas de realidade virtual e aumentada. Recife: Ed. Universitária da UFPE.

[15] Nayron Medeiros Soares et al. 2017. Terapia baseada em realidade virtual usando o Leap Motion Controller para reabilitação do membro superior após acidente vascular cerebral. Scientia Medica, v. 27, n. 2.

[16] Gabriel Fernandes Cyrino et al. 2019. HarpyGame: um jogo sério customizável com interface multimodal para reabilitação de indivíduos pós-AVE.

[17] Adriana Rocha Vidal et al. 2011. Teste funcional sistemático estendido: uma contribuição na aplicação de critérios de teste caixa-preta.

[18] Paulo Alexandre Bressan et al. 2003. Visualização de Alto Desempenho: Utilizando componentes convencionais. Porto Alegre: Editora SBC.
SVR2021' 18, October, 2021, Gramado, Rio Grande do Sul, Brazil 